\title{
Multi-glycoside of Tripterygium wilfordii Hook. f. attenuates glomerulosclerosis in a rat model of diabetic nephropathy by exerting anti-microinflammatory effects without affecting hyperglycemia
}

\author{
WEI WU ${ }^{1,2^{*}}$, JING-JING YANG ${ }^{1 *}$, HAI-MING YANG ${ }^{2}$, MENG-MENG HUANG $^{3}$, QI-JUN FANG ${ }^{2}$, \\ GE SHI $^{1}$, ZHI-MIN MAO ${ }^{1}$, WEN-BEI HAN ${ }^{1}$, SHAN-MEI SHEN ${ }^{4 * *}$ and YI-GANG WAN ${ }^{2 * *}$

\begin{abstract}
${ }^{1}$ Department of Traditional Chinese Medicine, Nanjing Drum Tower Hospital Clinical College of Traditional Chinese and Western Medicine, Nanjing University of Chinese Medicine; ${ }^{2}$ Department of Traditional Chinese Medicine,

Nanjing Drum Tower Hospital, The Affiliated Hospital of Nanjing University Medical School;

${ }^{3}$ Key Laboratory of New Drug Delivery System of Chinese Materia Medica, Jiangsu Provincial Academy of Chinese Medicine; ${ }^{4}$ Department of Endocrinology, Nanjing Drum Tower Hospital, The Affiliated Hospital of Nanjing University Medical School, Nanjing, Jiangsu 210008, P.R. China
\end{abstract}

Received August 2, 2016; Accepted July 10, 2017

DOI: $10.3892 / \mathrm{ijmm} .2017 .3068$

\begin{abstract}
Multi-glycoside of Tripterygium wilfordii Hook. f. (GTW) has been proven to be clinically effective in relieving microinflammation in patients with early diabetic nephropathy (DN). However, the therapeutic mechanisms involved in vivo remain unclear. In the process of early DN, microinflammation and activation of p38 mitogen-activated protein kinase (MAPK) and canonical nuclear factor (NF)- $\kappa \mathrm{B}$ signaling pathways are the important mechanisms by which hyperglycemia contributes to glomerulosclerosis (GS). Therefore, this study aimed to examine the ameliorative effects of GTW on GS, and then to clarify its anti-microinflammatory mechanisms by inhibiting p38 MAPK and NF- $\kappa$ B signaling activities in the kidney. All rats were divided into 4 groups: the sham group, the sham + GTW group, the vehicle group and the GTW group. The suitable dose of GTW and vehicle were daily administered for 8 weeks after the induction of
\end{abstract}

Correspondence to: Dr Yi-Gang Wan, Department of Traditional Chinese Medicine, Nanjing Drum Tower Hospital, The Affiliated Hospital of Nanjing University Medical School, 321 Zhongshan Road, Nanjing, Jiangsu 210008, P.R. China

E-mail:wyg68918@sina.com

Dr Shan-Mei Shen, Department of Endocrinology, Nanjing Drum Tower Hospital, The Affiliated Hospital of Nanjing University Medical School, 321 Zhongshan Road, Nanjing, Jiangsu 210008, P.R. China E-mail: shanmeishen@126.com

\section{${ }^{* * * *}$ Contributed equally}

Key words: multi-glycoside of Tripterygium wilfordii Hook. f., microinflammation, glomerulosclerosis, p38 mitogen-activated protein kinase signaling pathway, nuclear factor- $\mathrm{\kappa B}$ signaling pathway, diabetic nephropathy
DN by unilateral nephrectomy combined with intraperitoneal injections of streptozotocin (STZ). The general status of the rats, biochemical parameters, renal histological changes and macrophages in glomeruli, as well as expression of the key proteins in the p38 MAPK and canonical NF- $\kappa \mathrm{B}$ signaling pathways and inflammatory cytokines including tumor necrosis factor (TNF)- $\alpha$, interleukin (IL)-1 $\beta$ and transforming growth factor (TGF)- $\beta 1$ in the kidney were examined, respectively. The results revealed that, GTW improved the general condition and biochemical parameters of the rats, but did not lower blood glucose; GTW attenuated GS and suppressed glomerular microinflammation including the infiltration of $\mathrm{ED}^{+}$cells in glomeruli and the protein overexpression of TNF- $\alpha$, IL- $1 \beta$ and TGF- $\beta 1$ in the kidney; GTW inhibited the protein overexpression of key signaling molecules of p38 MAPK and canonical NF- $\kappa$ B pathways in the kidney including phosphorylated p38 MAPK, phosphorylated inhibitor protein I $\mathrm{B}$ and $\mathrm{NF}-\kappa \mathrm{B}$ (p65). On the whole, we expounded that GTW, as a natural regulator in vivo, alleviates GS without affecting hyperglycemia, by exerting antimicroinflammatory effects, including reducing macrophage infiltration in glomeruli, suppressing TNF- $\alpha$, IL- $1 \beta$ and TGF- $\beta 1$ overexpression in the kidney and inhibiting p38 MAPK and $\mathrm{NF}-\kappa \mathrm{B}$ signaling activities.

\section{Introduction}

In recent years, accumulating evidence in both the clinic and in experimental animal models have shown that microinflammation characterized by inflammatory cell proliferation, inflammatory cytokine overexpression and subsequent extracellular matrix (ECM) expansion in glomeruli is a common pathway for the progression of diabetic nephropathy (DN). Anti-microinflammatory strategies may thus offer approaches of great interest for early DN patients $(1,2)$. Utimura et al (3) reported that mycophenolate mofetil (MMF), 
an anti-inflammatory drug, inhibited glomerular macrophage infiltration and improved glomerulosclerosis (GS) in streptozotocin (STZ)-induced and uninephrectomized diabetic rats. Correspondingly, pentoxifylline (PTF), which possesses significant anti-inflammatory properties, has recently been proven to reduce urinary protein excretion in diabetic subjects, both with normal renal function and with renal insufficiency. Moreover, these beneficial effects are related to a reduction in the concentration of tumor necrosis factor (TNF)- $\alpha$, one of the most important pro-inflammatory cytokines $(4,5)$. Therefore, these findings also indicate that inhibition of microinflammation may be a therapeutic target and a pharmacological mechanism for protecting glomerular lesions in $\mathrm{DN}$.

It is well-known that microinflammation is regulated by inflammation-associated signaling pathways. The p38 mitogen-activated protein kinase (MAPK) signaling pathway becomes activated by inflammation stress-related signals including pro-inflammatory cytokines such as TNF- $\alpha$, interleukin (IL)-1, IL-6 and IL-18 through phosphorylation mediated by upstream kinases. In turn, phosphorylated p38 MAPK (p-p38 MAPK), as an important active p38 MAPK signaling molecule, enters the nucleus, as well as controls the expression of a variety of downstream inflammatory factors and inflammatory mediators, which lead to the promotion of renal inflammatory response that exacerbates glomerular damage $(2,6)$. For these reasons, by modulating the expression of p-p38 MAPK, transforming growth factor (TGF)- $\beta$ and nuclear factor (NF)- $\kappa \mathrm{B}$, it is possible to block inflammatory signaling pathway reactions and ameliorate relevant inflammatory injuries in the kidney.

Multi-glycoside of Tripterygium wilfordii Hook. f. (GTW) is a stable glycoside extracted from Tripterygium wilfordii Hook. f. (TWHF), also known as 'Lei Gong Teng' (the local name in China), which is a natural anti-inflammatory phytomedicine used for various autoimmune and inflammatory diseases in Eastern and Southern China, America, Korea and Japan (7-9). GTW, as a Chinese traditional patented medicine, has been approved by the China State Food and Drug Administration (Z32021007) for the routine treatment of glomerulonephritis and rheumatoid arthritis $(10,11)$. For the past 30 years, GTW at the dose of $60 \mathrm{mg}$ /day has been proven to be clinically effective in reducing proteinuria and hematuria by suppressing renal microinflammation, GS and podocyte injury in several types of human chronic kidney disease (CKD) (12-14). In addition, a single central study in China found that a large oral dose of GTW (120 mg/day) reduced proteinuria and alleviated renal dysfunction in $65 \mathrm{DN}$ patients at early and mid-term stages (15). More importantly, the therapeutic mechanisms of GTW and its main bioactive component, triptolide, may act by reducing macrophage proliferation in glomeruli (16-18). Despite this, its pharmacological mechanistic link in vivo between anti-microinflammatory effects and the protection against GS related to diabetes remains to be elucidated.

Hence, in this study, we examined the ameliorative effects of GTW on GS, using a modified STZ-induced DN rat model. We then clarified the anti-microinflammatory mechanisms in vivo of GTW by inhibiting the activation of inflammatory signaling pathways and the overexpression of inflammatory factors in the kidney. These results may provide a novel and effective therapeutic method for early stage DN patients.

\section{Materials and methods}

GTW quantity control. GTW purchased from Jiangsu Meitong Pharmaceutical Co., Ltd. (Taizhou, China) was composed of extracts from TWHF. One tablet contained $10 \mathrm{mg}$ of GTW. The extraction method and productive process of GTW are both subjected to strict quality control, and the main components are subjected to standardization (19). In addition, GTW is not only manufactured as granules after dynamic cycle extraction and concentration by evaporating and spray drying, but is also monitored for the absence of contaminants (heavy metals, pesticides, hormone and mycotoxins) prior to formulation. In this study, GTW (batch no. 150206) was dissolved in distilled water (GTW suspension) and stored at $4^{\circ} \mathrm{C}$ before use.

The quality of GTW was examined with fingerprint analysis by high-performance liquid chromatography (HPLC) in Jiangsu Province Academy of Chinese Medicine (Nanjing, China). As shown in Fig. 1, the known bioactive components including triptolide $\left(\mathrm{C}_{20} \mathrm{H}_{24} \mathrm{O}_{6}\right.$; CAS, 38748-32-2), triptonide $\left(\mathrm{C}_{20} \mathrm{H}_{22} \mathrm{O}_{6}\right.$; CAS, 38647-11-9), wilforgine $\left(\mathrm{C}_{41} \mathrm{H}_{47} \mathrm{NO}_{19}\right.$; CAS, 37239-47-7), wilforine $\left(\mathrm{C}_{43} \mathrm{H}_{49} \mathrm{O}_{18}\right.$; CAS, 11088-09-8), tripterine $\left(\mathrm{C}_{29} \mathrm{H}_{38} \mathrm{O}_{4}\right.$; CAS, 34157-83-0) and wilforlide $\mathrm{A}$ $\left(\mathrm{C}_{30} \mathrm{H}_{46} \mathrm{O}_{3}\right.$; CAS, 84104-71-2) $(20,21)$ (Fig. 1A) in 5 batches exhibited high stability.

Animals, drugs and reagents. All experiments were performed using male Sprague-Dawley (SD) rats weighing from 180 to $200 \mathrm{~g}$, purchased from the Animal Center of Nanjing Military District General Hospital (Nanjing, China). The surgical procedures and experimental protocol were approved by the Animal Ethics Committee of Nanjing University Medical School. STZ was purchased from Sigma-Aldrich (St. Louis, MO, USA). Antibodies against TGF- $\beta 1$, TNF- $\alpha$, IL- $1 \beta$ and horseradish peroxidase (HRP)-labeled IgG were purchased from Abcam (Cambridge, UK). Antibodies against p38 MAPK, p-p38 MAPK, p-IкB and NF- $\kappa$ B (p65) were purchased from Cell Signaling Technology, Inc. (Beverly, MA, USA). The antibody against glyceraldehyde 3-phosphate dehydrogenase (GAPDH) was obtained from Bioworld Technology, Inc. (Louis Park, MN, USA).

Experimental protocol. The experimental procedure is illustrated in Fig. 2. The DN model was established as described in our previous study (22). Twenty rats were divided into 4 groups: the sham group (sham operation + distilled water), the sham + GTW group (sham operation + GTW), the vehicle group (DN + distilled water) and the GTW group (DN + GTW). In the clinic, GTW at a dose of $60 \mathrm{mg} /$ day is used to treat a patient weighing $60 \mathrm{~kg}(11,15)$, which is equivalent to $50 \mathrm{mg} / \mathrm{kg} /$ day in rats. Following the second injection of STZ, the GTW suspension was administered to the rats in the 2 GTW-treated groups by gastric gavage once a day for 8 weeks, while rats in the vehicle and sham group were treated with $2 \mathrm{ml}$ distilled water. Eight weeks after administration, all rats were anesthetized and sacrificed through cardiac puncture. Blood samples and kidneys were collected for detection of various indicators.

General status and biochemical parameters of the rats. Energy level, diet, water intake, fur color and activities of the rats in each group were observed daily. Body weight (BW), blood 


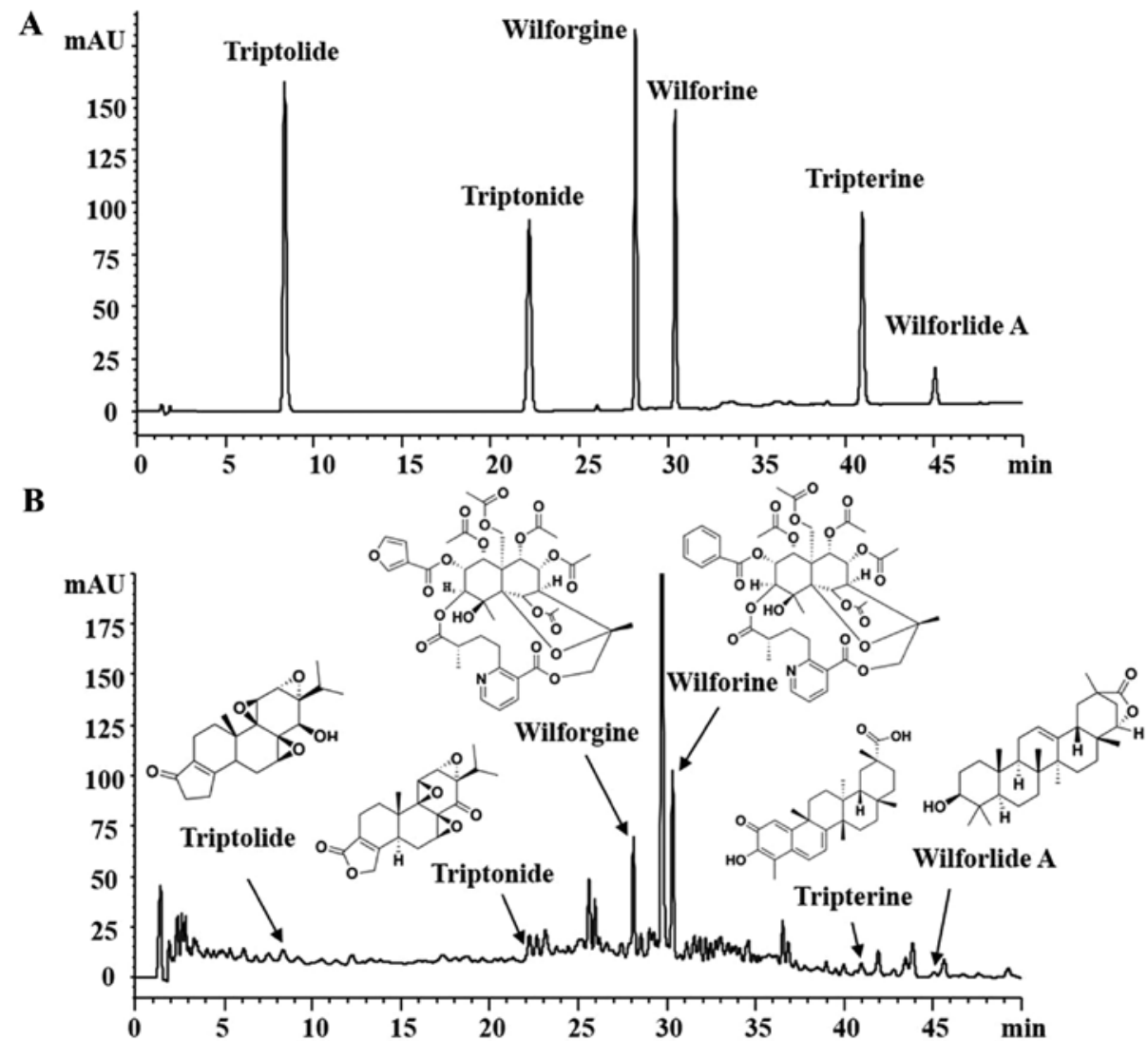

Figure 1. (A) Chromatograms of mixed standards and (B) samples of Tripterygium wilfordii Hook. f. (GTW).

glucose (BG) and urinary albumin (UAlb) of the rats were detected respectively before and every 2 weeks after modeling. The right kidney of rats in each group was removed and weighed after cardiac puncture. At the end of week 8 after drug-intervention, the rats were anesthetized and blood samples $(2 \mathrm{ml})$ were drawn from the heart. Indices including serum creatinine (Scr), blood urea nitrogen (BUN), serum alanine transaminase (ALT) and serum aspartate transaminase (AST) were detected.

Renal histomorphometry.Periodic acid-Schiff(PAS) staining, Masson staining and electron microscopic assessment were performed as previously described (22). Cell numbers, ECM and collagen rates in the glomerulus were calculated with Image-Pro Plus 6.0 software (Media Cybernetics, Inc., Rockville, MD, USA). The results were confirmed by a professional pathologist.

Immunohistochemistry. Macrophages were detected in 4- $\mu \mathrm{m}$ thick paraffin-embedded renal sections. For immunostaining of macrophages, monoclonal anti-ED1 antibody (AbD Serotec, Oxford, UK) was used. Quantitative analysis of ED1 ${ }^{+}$cells was performed in a blinded fashion and expressed as cells/ glomerular cross section (gcs). The results were also confirmed by the pathologist professional.

Semi-quantitative western blot analysis. Western blot analysis was performed as previously described (22). Renal tissues from the rats were isolated with phosphate-buffered saline (PBS) including protease inhibitors (PI) and sequentially solubilized

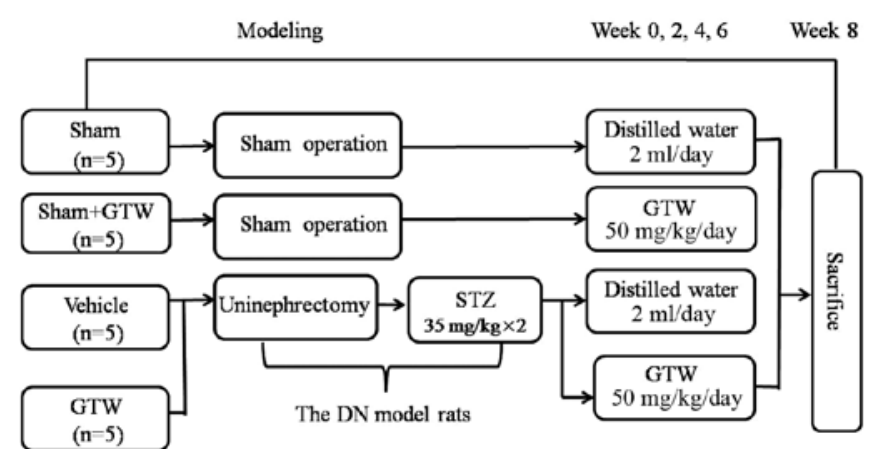

Figure 2. Experimental procedure.

with $1 \%$ Triton X-100, RIPA buffer $(0.1 \%$ SDS, $1 \%$ sodium deoxycholate, $1 \%$ Triton $\mathrm{X}-100,0.15 \mathrm{~mol} / 1 \mathrm{NaCl}$ and $0.01 \mathrm{~mol} / 1$ ethylenediaminetetraacetic acid (EDTA) in $0.025 \mathrm{~mol} / 1 \mathrm{Tris}-\mathrm{HCl}$, $\mathrm{pH}$ 7.2) with PI, and separated into Triton X-100-soluble (T), RIPA-soluble (R) and RIPA-insoluble (S) fractions. The RIPAIinsoluble fraction was solubilized with SDS-PAGE sample buffer (2\% SDS, $10 \%$ glycerol, and 5\% 2-mercaproethanol in $0.0625 \mathrm{~mol} / \mathrm{l}$ Tris-HCl, $\mathrm{pH}$ 6.8) (Sfractions). Equal amounts of theses equentially solubilized fractions were subjected to SDS-PAGE with 7.5 or $10 \%$ acrylamide gel, and transferred onto a PVDF membrane (Bio-Rad, Hercules, CA, USA) by electrophoretic trans-blotting for $30 \mathrm{~min}$ using Trans-Blot SD (Bio-Rad). After blocking with BSA, the strips of membrane were exposed to anti-p38 MAPK, p-p38 MAPK, ІкB, p-ІкB, NF- $\kappa \mathrm{B}$ (p65), TGF- $\beta 1$, TNF- $\alpha$, IL- $1 \beta$ and GAPDH antibodies, 
A
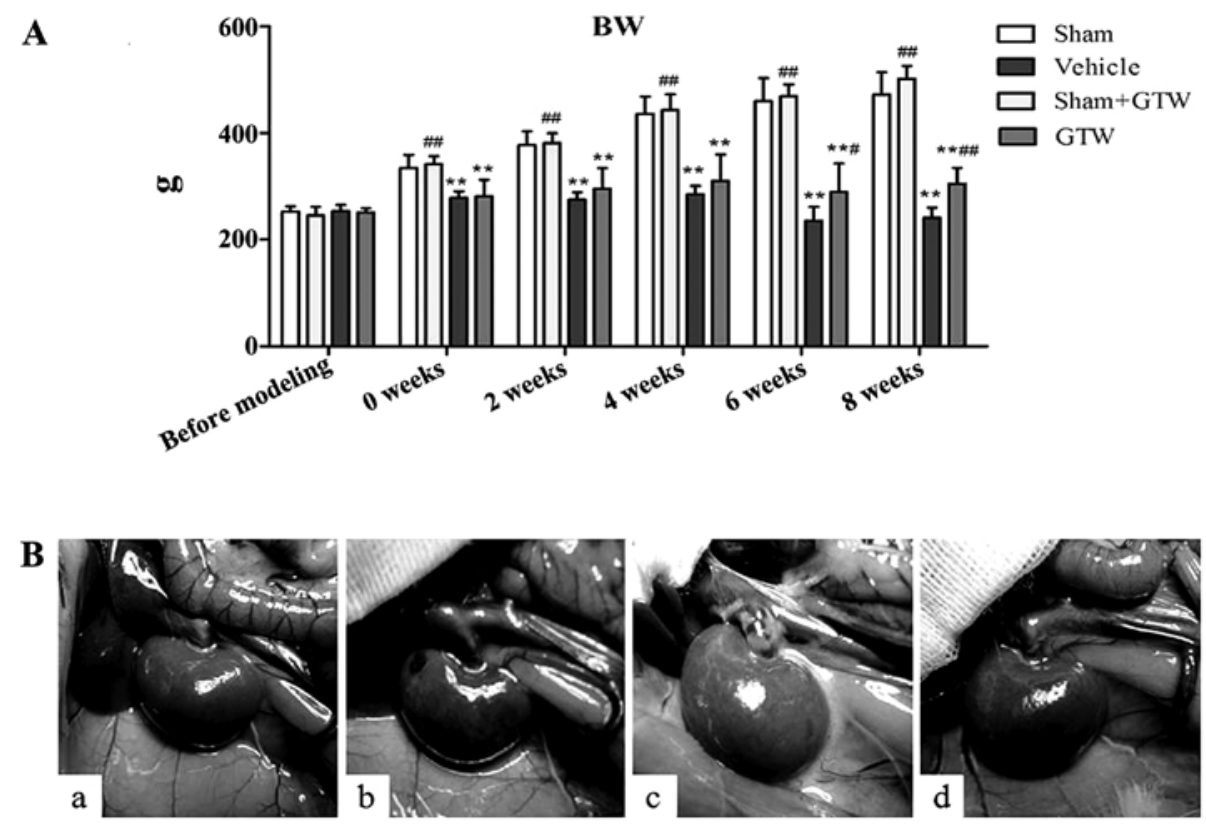

C

KW

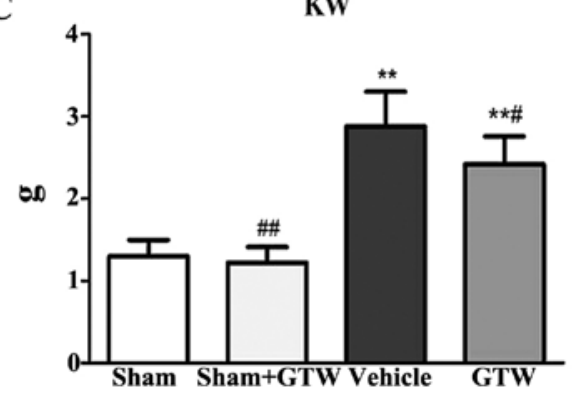

Figure 3. Effects of Tripterygium wilfordii Hook. f. (GTW) on changes in (A) body weight (BW), (B) renal appearance and (C) kidney weight (KW) in the 4 groups: (a) the sham group, (b) the sham + GTW group, (c) the vehicle group and (d) the GTW group. Data are expressed as mean \pm SE. ${ }^{* *} \mathrm{P}<0.01 \mathrm{vs}$. the sham group; ${ }^{~} \mathrm{P}<0.05,{ }^{\# \#} \mathrm{P}<0.01$ vs. the vehicle group at every 2 weeks or week 8 after drug-intervention.

respectively. They were washed and incubated with peroxidaseconjugated secondary antibodies for $1 \mathrm{~h}$ at room temperature. The bands were visualized by employing analkaline phosphatase chromogen kit (5-bromo-4-chloro-3-indolil phos-phate p-toluidine salt/nitro blue tetrazolium; Biosynth AG, Staad, Switzerland). The density of the positive bands was quantitated by Densitograph (ATTO, Tokyo, Japan). This procedure was carried out 3 times. The ratio of the densitometric signal of the molecules examined to that of GAPDH was determined. Data are shown as ratios relative to control findings and expressed as mean \pm SE. of 3 independent experiments.

Statistical analysis. The differences among groups were analyzed by one-way analysis of variance (ANOVA), and LSD method was used for multiple comparison. Qualitative data were analyzed using Fisher's exact test as indicated. $\mathrm{P}<0.05$ was considered statistically significant.

\section{Results}

GTW improves the general condition and biochemical parameters of the rats, but does not lower BG. During the experiment, the rats in the vehicle group showed increased diet, water intake and urine volume, low activity, dull fur and BW loss at different degrees. BW of rats in the vehicle group increased slowly from week 2. After GTW-intervention, BW of rats in the GTW group increased gradually. At the end of week 6 and 8, BW of rats in the GTW group was obviously higher than that in the vehicle group, and the differences were statistically significant (Fig. 3A). After sacrifice, we found that renal appearance in the sham and sham + GTW groups was moderated and crimson, while the kidneys in the vehicle group were swollen and pale. The kidneys of rats treated with GTW were significantly ameliorated, with less swelling and ischemia (Fig. 3B). In addition, kidney weight $(\mathrm{KW})$ of rats in the vehicle group was obviously higher than that in the sham group. While KW of rats in the GTW group declined, and the differences were statistically significant compared with the vehicle group, but they were still higher than that in the sham group (Fig. 3C).

Next, we investigated the effects of GTW on BG and UAlb in the 4 rat groups. During the entire course, BG of rats in the sham and sham + GTW groups remained at low levels, while it increased in the rats of the vehicle group after two injections of STZ for $72 \mathrm{~h}$, and remained at a high level (random BG, $20.3 \pm 1.72 \mathrm{mmol} / \mathrm{l}$ ) by the consecutive intervention with Novolin N. Moreover, notably, BG of rats in the GTW group also remained high, and the differences were statistically significant compared with the sham group (Fig. 4A). The level 

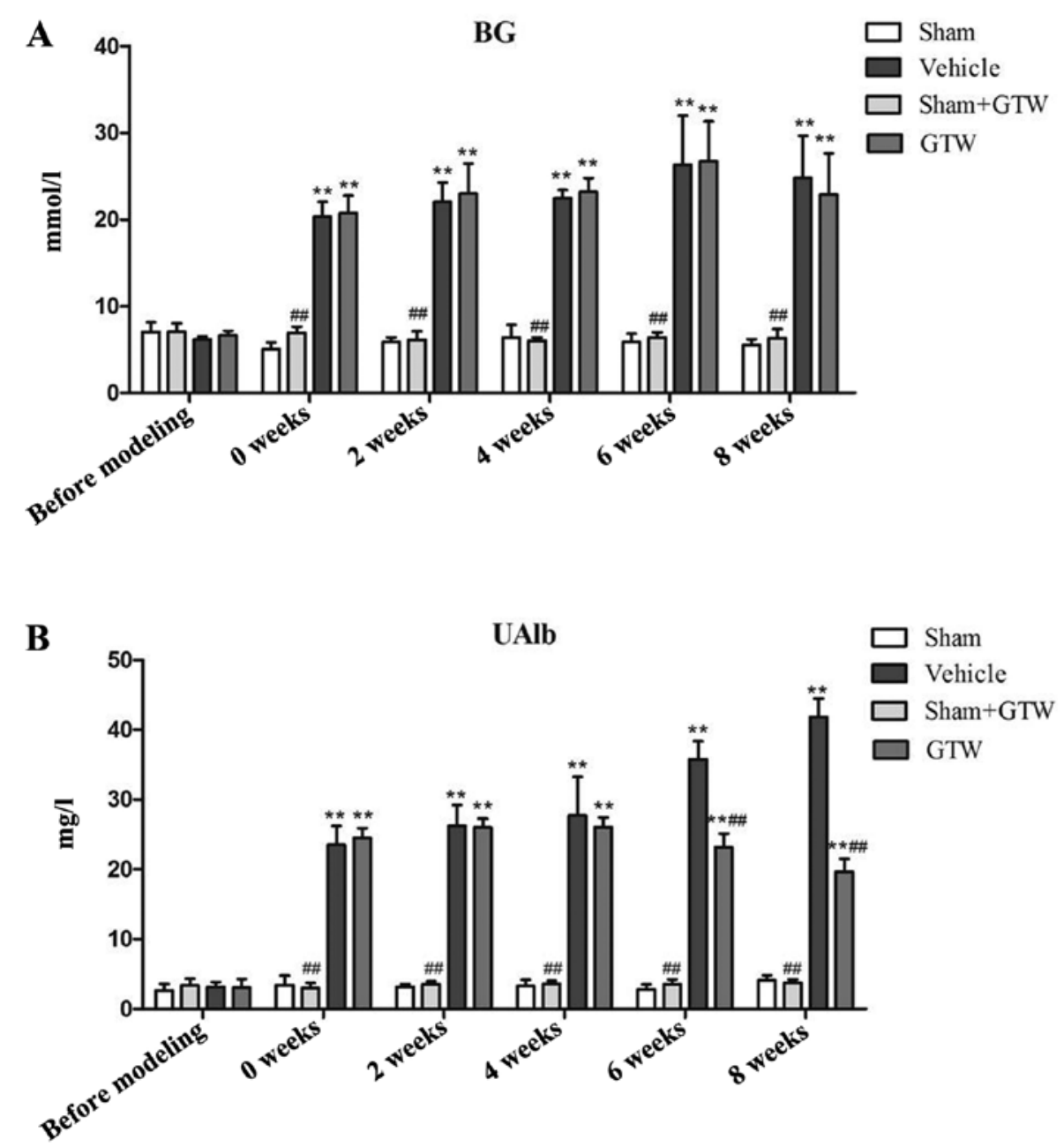

Figure 4. Effects of Tripterygium wilfordii Hook. f. (GTW) on changes in (A) blood glucose (BG) and (B) urinary albumin (UAlb) in the 4 groups. Data are expressed as mean $\pm \mathrm{SE}$. ${ }^{* *} \mathrm{P}<0.01$ vs. the sham group; ${ }^{\# \#} \mathrm{P}<0.01$ vs. the vehicle group at every 2 weeks.
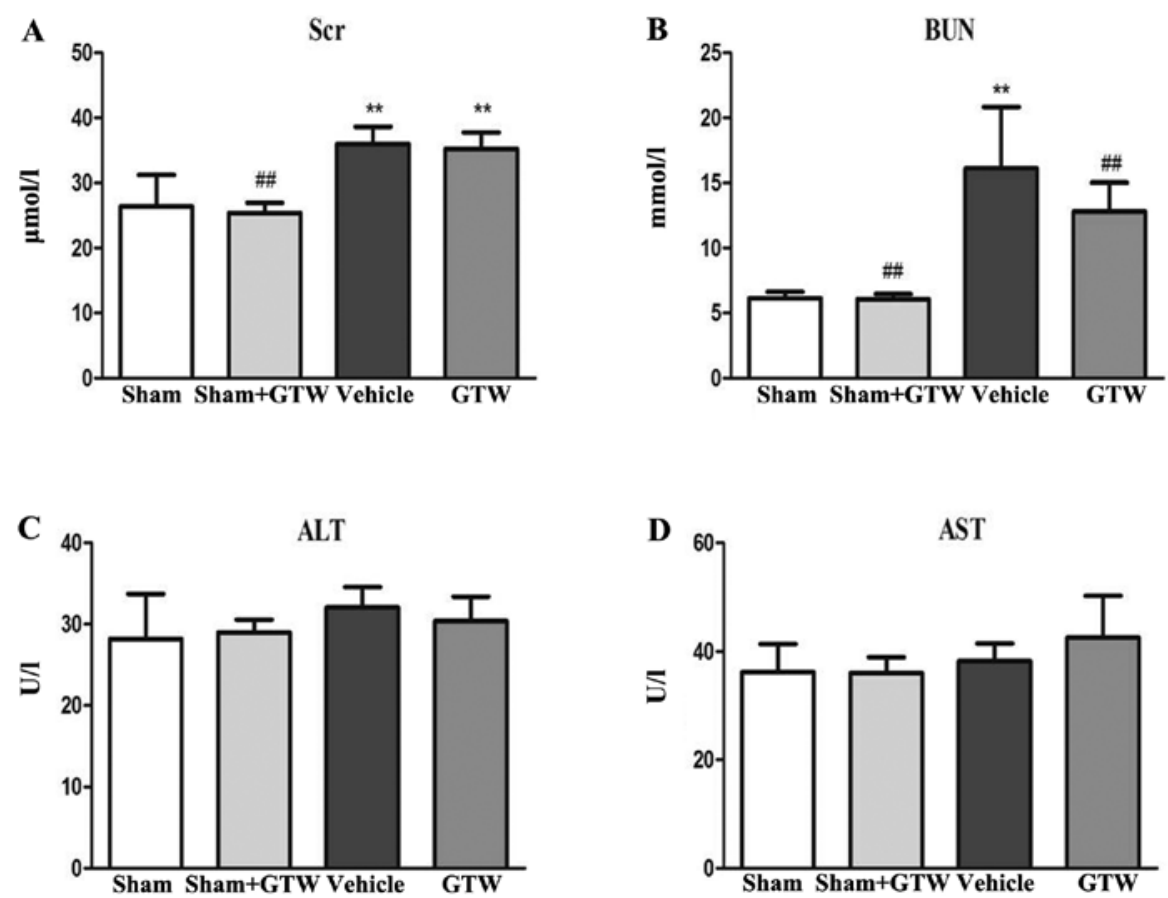

Figure 5. Effects of Tripterygium wilfordii Hook. f. (GTW) on changes in (A) serum creatinine (Scr), (B) blood urea nitrogen (BUN), (C) serum alanine transaminase (ALT) and (D) serum aspartate transaminase (AST) in the 4 groups. Data are expressed as mean $\pm \mathrm{SE}$. ${ }^{* * *} \mathrm{P}<0.01 \mathrm{vs}$. the sham group; ${ }^{* \#} \mathrm{P}<0.01$ vs. the vehicle group at week 8 after drug-intervention. 

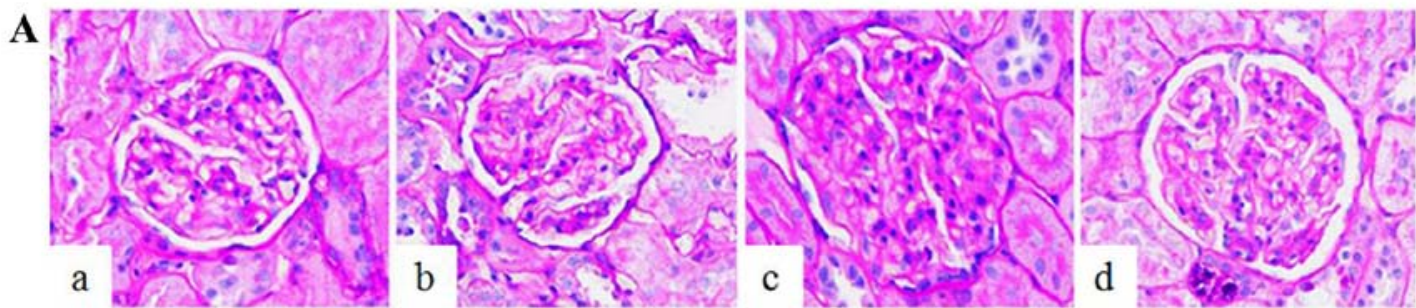

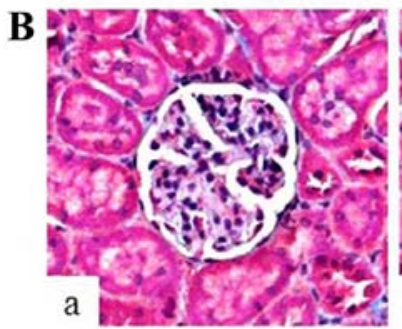

Cell number/glomeruli

C

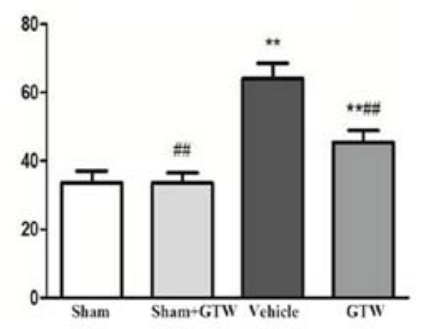

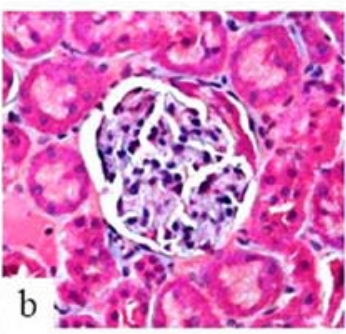

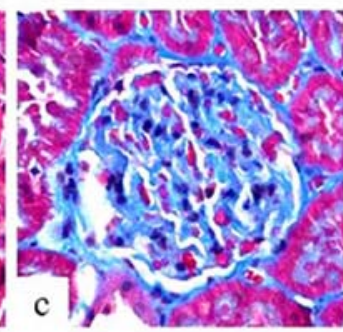

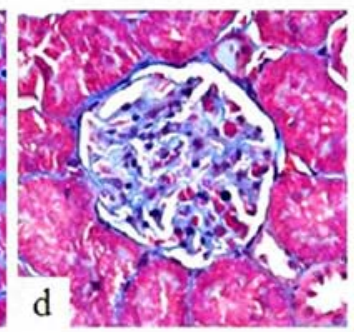

Collagen/glomerular area
D

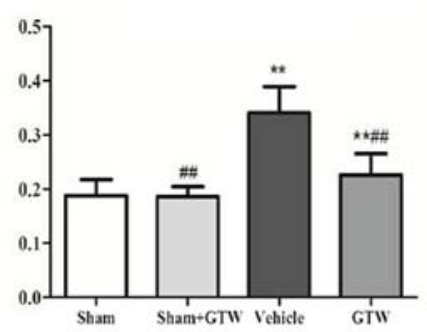

$\mathbf{E}$

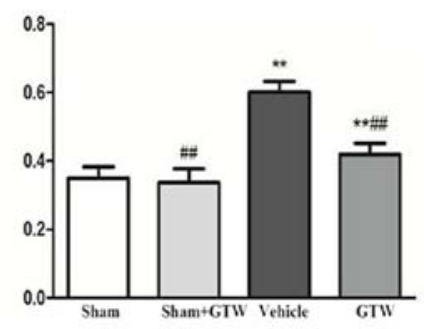

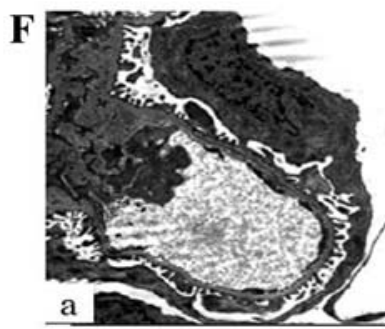
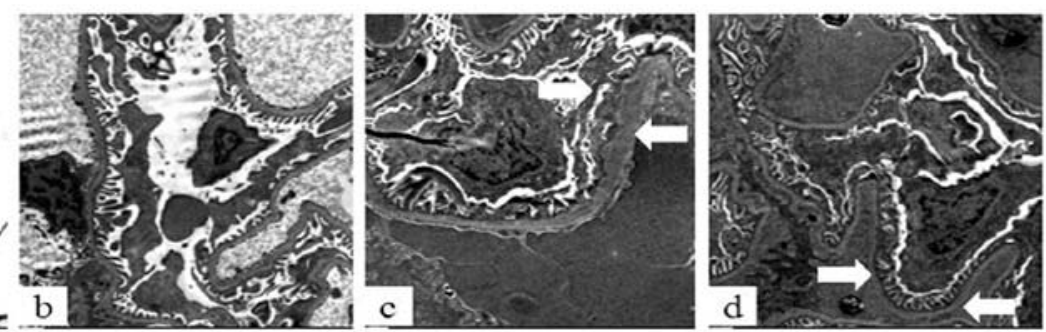

Figure 6. Effects of Tripterygium wilfordii Hook. f. (GTW) on the glomerular morphological changes in the 4 groups. (A) Light microscopy, periodic acid-Schiff (PAS) (magnification, x400). (B) Light microscopy, Masson staining (magnification, x400). (C) The number of glomerular cells. (D) The rate of extracellular matrix (ECM)/glomerular area. (E) The rate of collagen/glomerular area. (F) Electron microscopy, x1,700. The arrows in (F-c) indicate foot process effacement and glomerular basement membrane (GBM) thicking respectively. The arrows in (F-d) indicate the recovered foot process and GBM respectively. (a) Sham group, (b) sham + GTW group, (c) vehicle group and (d) GTW group. Data are expressed as mean \pm SE. ${ }^{* *} \mathrm{P}<0.01$ vs. the sham group; ${ }^{\#} \mathrm{P}<0.01 \mathrm{vs}$. the vehicle group at week 8 after drug-intervention.

of UAlb in each group was low before modeling, but rapidly increased 2 weeks after modeling, and constantly increased with the extension of time in the vehicle group. At the end of week 8 , UAlb in the vehicle group reached an abnormal level $(41.82 \pm 2.61 \mathrm{mg} / \mathrm{l})$, which was significantly higher than that in the sham and sham + GTW groups. After GTW-intervention, the level of UAlb was significantly decreased in the GTW group at week 8 , and the difference was statistically significant compared with the vehicle group (Fig. 4B).

Thirdly, we examined the effects of GTW on blood biochemical parameters including Scr, BUN, ALT and AST in the 4 rat groups. At the end of week 8 after GTW-intervention, serum ALT and AST in each group had no obvious changes, but Scr and BUN in the vehicle group were increased, and the differences were statistically significant respectively compared with the sham and sham + GTW groups. After GTW treatment, the levels of Scr and BUN were decreased, but the differences were not statistically significant compared with the vehicle group (Fig. 5).

GTW attenuates GS. We observed glomerular morphological changes by light microscopy in the 4 rat groups. In the sham and sham + GTW groups, glomerular capillary loops were well-opened and tubular epithelial cells were arranged in order. Mesangial tissues had hyperplasia in the vehicle group including glomerular mild-hypertrophy, capillary loop area reduction, glomerular cell proliferation, ECM expansion and collagen deposition. With GTW treatment, the injurious glomerular morphological changes were obviously attenuated. When compared with the sham group, cell numbers, rate 
A

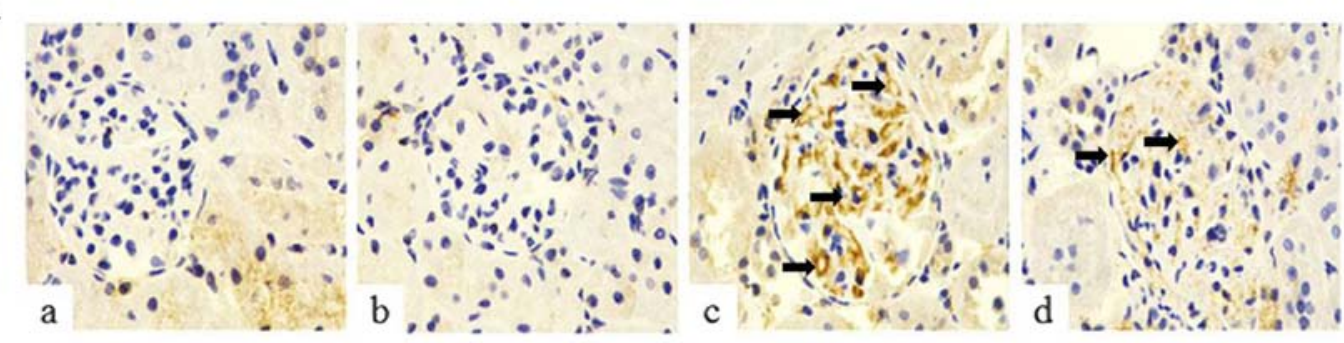

B

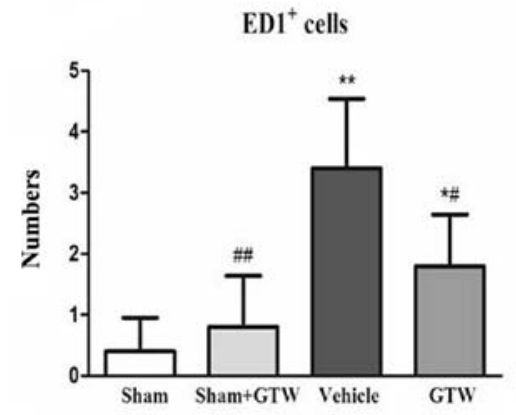

D

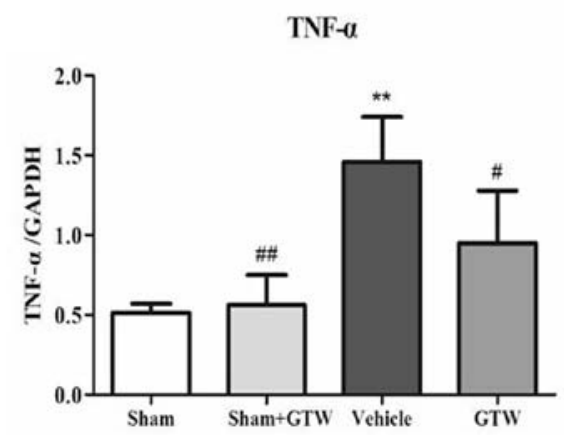

C

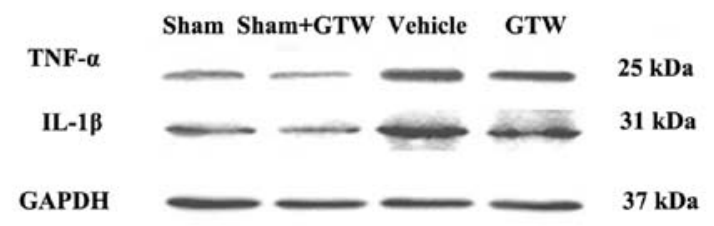

E

IL-1 $1 \beta$

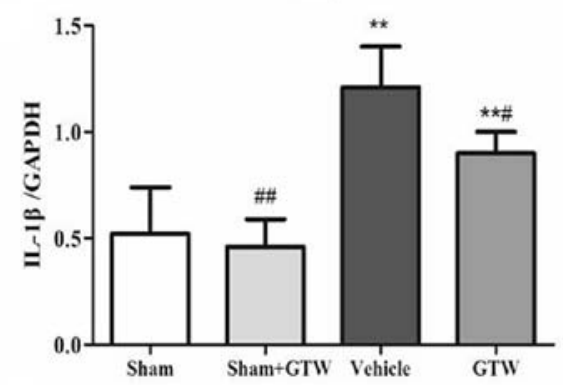

Figure 7. Effects of Tripterygium wilfordii Hook. f. (GTW) on the infiltration of ED1 $1^{+}$cells in glomeruli and the protein expression of tumor necrosis factor (TNF)- $\alpha$ and interleukin (IL)-1 $\beta$ in the kidney in the 4 groups. (A and B) Immunohistochemical analysis and numbers of infiltrative ED1 ${ }^{+}$cells. (a) Sham group, (b) sham + GTW group, (c) vehicle group and (d) GTW group. (C-E) Western blot analysis and quantitative analysis of TNF- $\alpha$ and IL-1 $\beta$ protein expression. Data are expressed as mean $\pm \mathrm{SE} .{ }^{* *} \mathrm{P}<0.01$ vs. the sham group; ${ }^{\#} \mathrm{P}<0.05,{ }^{\# \#} \mathrm{P}<0.01$ vs. the vehicle group at week 8 after drug-intervention.

of ECM and collagen area in glomerulus were significantly increased in the vehicle group respectively, but decreased in the GTW group. However, no significant differences in glomerular morphological changes were found between the sham and sham + GTW groups (Fig. 6A-E). We measured podocytic morphological characteristics and glomerular basement membrane (GBM) thickness by electron microscopy in the 4 rat groups. Unexpectedly, the injurious morphological changes in podocytes and GBM including foot process effacement and GBM thicking in the vehicle group were significantly alleviated in the GTW group (Fig. 6F).

GTW suppresses glomerular microinflammation. We determined the number of ED1 ${ }^{+}$cells, a marker of total macrophages in glomeruli. By means of immunohistochemical staining, the number of $\mathrm{ED}^{+}$cells in glomeruli was increased notably in the vehicle group, while the number declined significantly in the GTW group (Fig. 7A and B). No obvious infiltrated macrophages in the glomeruli were detected in the sham and sham + GTW groups. We further investigated the protein expression of inflammatory cytokines in the kidney including
TNF- $\alpha$ and IL-1 $\beta$ in 4 rat groups. Western blot analysis of renal tissues revealed that the protein expression of TNF- $\alpha$ and IL-1 $\beta$ was markedly upregulated in the vehicle group. Compared with the vehicle group, the protein overexpression of TNF- $\alpha$ and IL- $1 \beta$ in the kidney was significantly reduced in the GTW group (Fig. 7C-E).

GTW inhibits activation of p38 MAPK and canonical $N F-\kappa B$ signaling pathways in the kidney. Semi-quantitative western blot analysis showed that the expression level of p-p38 MAPK, $\mathrm{p}-\mathrm{I} \kappa \mathrm{B}, \mathrm{NF}-\kappa \mathrm{B}(\mathrm{p} 65)$ and TGF- $\beta 1$ at the protein levels in the vehicle group was significantly higher than those in the sham group. Compared with the vehicle group, the protein overex-

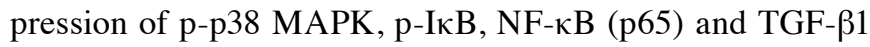
was significantly reduced in the GTW group (Fig. 8).

\section{Discussion}

In the present study, we demonstrated that GTW, a natural anti-inflammatory phytomedicine, can improve GS and glomerular microinflammation in vivo. Nevertheless, GTW had no 
A
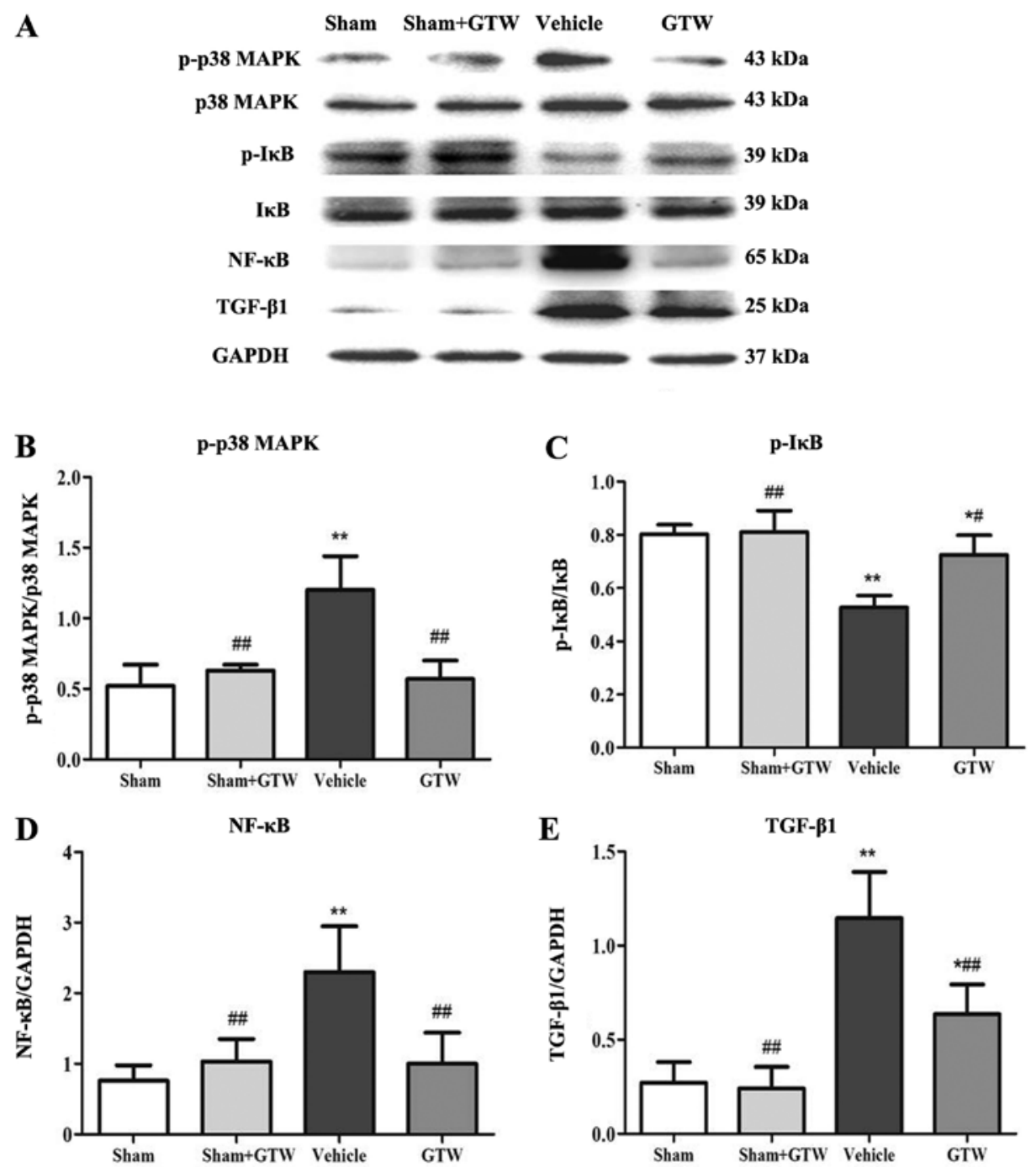

Figure 8. Effects of Tripterygium wilfordii Hook. f. (GTW) on the protein expression of p-p38 mitogen-activated protein kinase (MAPK), p38 MAPK, p-IкB, IкB, nuclear factor (NF)- $\mathrm{\kappa B}(\mathrm{p} 65)$ and transforming growth factor (TGF)- $\beta 1$ in the kidney in the 4 rat groups. (A) Western blot analysis of p-p38 MAPK, p38 MAPK,

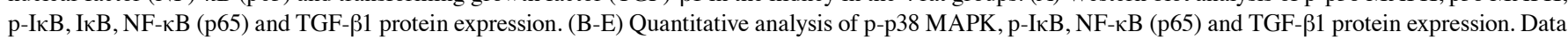
are expressed as mean $\pm \mathrm{SE}$. ${ }^{*} \mathrm{P}<0.05$ vs. the sham group; ${ }^{* *} \mathrm{P}<0.01$ vs. the sham group; ${ }^{* \prime \prime} \mathrm{P}<0.01$ vs. the vehicle group at week 8 after drug-intervention.

effect on hyperglycemia in these DN model rats. To clarify the therapeutic effects of phytomedicines on renal damage related with diabetes in vivo, it is necessary to establish an appropriate animal model that simulates human DN patients. In this study, we used a modified DN rat model by unilateral nephrectomy combined with STZ intraperitoneal injections with low doses of $35 \mathrm{mg} / \mathrm{kg} \mathrm{BW}$ at a $72-\mathrm{h}$ interval. Our results showed that these DN model rats not only had stable hyperglycemia and a certain degree of UAlb, but also had typical GS characteristics. Therefore, we believed that this DN rat model was conducive to discovering novel therapeutic phytomedicines for human DN.

It has been reported that microinflammation plays a pivotal role in promoting glomerular injury in several studies of human and experimental DN induced by STZ injection (23). During microinflammation in renal parenchyma, macrophages can be attracted/activated directly by mechanical stress and may produce pro-inflammatory cytokines such as TNF- $\alpha$, IL- 1 , IL-6 and IL-18, thus recruiting additional inflammatory cells that contribute to the propagation of inflammation and GS (24).
Hence, to investigate whether the anti-microinflammatory effects of GTW could be related to the improvement of GS in vivo, we examined the number of infiltrated $\mathrm{ED}^{+}$cells in glomeruli, a marker of total macrophages, and the protein expression of TNF- $\alpha$ and IL-1 $\beta$ in the kidney, representative inflammatory cytokines, at the end of week 8 after two STZ injections in the GTW and vehicle groups, respectively. Our results showed that macrophage infiltration, affecting almost exclusively glomeruli, was evident at the end of week 8 after modeling in the vehicle group. Furthermore, the intensity of macrophage infiltration in glomeruli and the levels of inflammatory cytokine expression in the kidney were strongly correlated with the injurious characteristics of GS in these DN model rats. In addition to these, GTW suppressed glomerular microinflammation together with the amelioration of GS. Consequently, GTW, as an anti-inflammatory phytomedicine, can alleviate GS in vivo, which is possibly connected with anti-microinflammatory action.

To the best of our knowledge, the therapeutic action in DN animal models of various anti-inflammatory agents such 
as thiazolidinediones, 1,25-dihydroxyvitamin $\mathrm{D}_{3}$, cilostazol, curcumin (23) and Huangkui capsule (22) are directly related to controlling activation of intracellular signaling pathways associated with renal microinflammation, in which p38 MAPK, Janus kinase/signal transducers and activators of transcription (JAK/STAT) and NF-кB-dependent pathways occupy the key positions in DN at the early stage. Thus, we proposed that regulating the activation of $\mathrm{p} 38 \mathrm{MAPK}$ and/or canonical NF- $\kappa \mathrm{B}$ pathways in this DN rat model are the successful means with which to identify anti-inflammatory mechanisms of GTW on attenuating GS in vivo. Our data clearly indicated that enhanced protein expression of p-p38 MAPK, p-ІкB, NF- $\kappa B$ (p65) and TGF- $\beta 1$ in the kidney were obviously detected in these DN model rats, concomitant with deterioration of GS induced by microinflammation. These results indicated that the p38 MAPK and canonical NF- $\kappa B$ pathways were activated in this DN rat model, and there is a strong causality between key signaling molecular overexpression and GS in vivo. More importantly, we also found that GTW simultaneously downregulated the protein overexpression of p-p38 MAPK, p-IкB, $N F-\kappa B(p 65)$ and TGF- $\beta 1$ in the kidney of these DN model rats. For these reasons, we suggest that GTW in vivo can attenuate GS by inhibiting p38 MAPK pathway activation and the release of NF- $\kappa B$ dimer p50/p65 in the canonical NF- $\kappa B$ pathway. Here, unfortunately, we could not assess whether GTW in vivo directly blocks the p38 MAPK signaling pathway based upon this DN rat model, without the signaling inhibitors. Further detailed analyses in vitro of p38 MAPK signaling molecules are needed to address this hypothesis.

Finally, we need to discuss three additional points. First, GTW, a natural anti-inflammatory phytomedicine, did not affect hyperglycemia in this DN rat model induced by STZ injection. We unavoidably think of the cause-and-effect of the relationship among hyperglycemia, microinflammation and GS. Some studies have shown that activation of the immune system and microinflammation are both involved in the pathogenesis of GS in DN and of course play an important role in vivo (25). Thus, we firmly believe that GTW has renoprotective action similar to other CKD models such as the anti-Thy1.1 glomerulonephritis rat model (26) and the adriamycin-induced GS rat model (27), completely independent of affecting hyperglycemia. Second, GTW has been proven clinically effective in suppressing microinflammation in various CKDs; however, its clinical application is often limited by side effects to the liver (28). To exclude the side effects of GTW on hepatic damage in this DN rat model, we compared the levels of serum ALT and AST in the 4 rat groups. Our results revealed that serum ALT and AST in the groups had no obvious changes, especially between the sham and sham + GTW groups (Fig. 5), thereby indicate that GTW at the suitable dose of $50 \mathrm{mg} / \mathrm{kg} \mathrm{BW}$ daily had no negative effect on liver function in these DN model rats. Third, although GTW affected the activation of the p38 MAPK and canonical NF- $\mathrm{kB}$ pathways in vivo, we could not draw a clear conclusion regarding the key molecular mechanisms of p38 MAPK and NF- $\kappa B$ pathways in this DN rat model. It may be worth pursing whether $\mathrm{p} 38 \mathrm{MAPK}$ at the upstream controls $\mathrm{NF}-\kappa \mathrm{B}$ transcriptional activation both in vivo and in vitro since NF- $\kappa \mathrm{B}$ is activated by a wide variety of stimuli such as cytokines, oxygen radicals, bacterial products and metabolic abnormalities, which are related with p38 MAPK activa- tion (29). A diabetic knockout mouse (30) and a special test of NF- $\mathrm{KB}$ (p65) DNA-binding activity (31) in the kidney should be necessary in future studies to clarify this point.

In conclusion, in the present study, we demonstrated that GTW, as a natural regulator in vivo, alleviated GS without affecting hyperglycemia using modified DN model rats, by exerting anti-microinflammatory effects, including reducing macrophage infiltration in glomeruli, suppressing TNF- $\alpha$, IL- $1 \beta$ and TGF- $\beta 1$ overexpression in the kidney and inhibiting p38 MAPK and NF- $\kappa B$ signaling activities. To the best of our knowledge, the findings of this study provide the first evidence in vivo that GTW directly contributes to the prevention of DN by exerting anti-microinflammatory effects.

\section{Acknowledgements}

This study was supported by two grants from the National Natural Science Foundation of China (81374030 and 81573903) (to Y.-G.W.) and a grant from Nanjing Medical Science and Technique Development Foundation (to W.W.). The authors thank Dr Xun-Yang Luo and Dr Le Zhang (Department of Laboratory Medicine, Nanjing Drum Tower Hospital, The Affiliated Hospital of Nanjing University Medicine School, Nanjing, China) for their technical assistance and instructions. The authors also thank Professor Jian Yao (Division of Molecular Signaling, Department of Advanced Biomedical Research, Interdisciplinary Graduate School of Medicine and Engineering, University of Yamanashi, Yamanashi, Japan) and Professor Yan Chen (Key Laboratory of New Drug Delivery System of Chinese Materia Medica, Jiangsu Provincial Academy of Chinese Medicine, Nanjing, China) for their helpful discussions and technical assistance.

\section{References}

1. Agrawal NK and Kant S: Targeting inflammation in diabetes: newer therapeutic options. World J Diabetes 5: 697-710, 2014.

2. Navarro-González JF, Mora-Fernández C, Muros de Fuentes M and García-Pérez J: Inflammatory molecules and pathways in the pathogenesis of diabetic nephropathy. Nat Rev Nephrol 7: 327-340, 2011.

3. Utimura R, Fujihara CK, Mattar AL, Malheiros DM, Noronha IL and Zatz R: Mycophenolate mofetil prevents the development of glomerular injury in experimental diabetes. Kidney Int 63: 209-216, 2003.

4. Navarro JF, Mora C, Muros M and García J: Additive antiproteinuric effect of pentoxifylline in patients with type 2 diabetes under angiotensin II receptor blockade: a short-term, randomized, controlled trial. J Am Soc Nephrol 16: 2119-2126, 2005.

5. Navarro-González JF, Mora-Fernández C, Muros de Fuentes M, Chahin J, Méndez ML, Gallego E, Macía M, del Castillo N, Rivero A, Getino MA, et al: Effect of pentoxifylline on renal function and urinary albumin excretion in patients with diabetic kidney disease: The PREDIAN trial. J Am Soc Nephrol 26: 220-229, 2015

6. Schieven GL: The p38alpha kinase plays a central role in inflammation. Curr Top Med Chem 9: 1038-1048, 2009.

7. Brinker AM, Ma J, Lipsky PE and Raskin I: Medicinal chemistry and pharmacology of genus Tripterygium (Celastraceae). Phytochemistry 68: 732-766, 2007.

8. Marks WH: Tripterygium wilfordii Hook F. versus sulfasalazine in the treatment of rheumatoid arthritis: a well-designed clinical trial of a botanical demonstrating effectiveness. Fitoterapia 82: 85-87, 2011.

9. Wan YG, Gu LB and Shimizu F: Mechanism of protective effects of effective components in Ttipterygium wilfordii Hook. f. on glomerulonephritis. Int J Clin Exp Med 207: 285-288, 2003 (In Japanese). 
10. Jiang M, Zha Q, Zhang C, Lu C, Yan X, Zhu W, Liu W, Tu S, Hou L, Wang C, et al: Predicting and verifying outcome of Tripterygium wilfordii Hook F. based therapy in rheumatoid arthritis: from open to double-blinded randomized trial. Sci Rep 5: 9700, 2015.

11. Leishi L: Clinical study of Tripterygium wilfordii Hook in treating glomerulonephritis (author's transl). Zhonghua Nei Ke Za Zhi 20: 216-220, 1981 (In Chinese).

12. Liu ZH, Li SJ, Wu Y, Zuo K, Wang B, Zeng CH and Li LS: Treatment of membranous nephropathy with Tripterygium wilfordii and steroid: a prospective randomized control trial. J Nephrol Dialy Transpl 18: 303-309, 2009 (In Chinese).

13. Ma R, Xu Y, Jiang W and Zhang W: Combination of Tripterygium wilfordii Hook $\mathrm{F}$ and angiotensin receptor blocker synergistically reduces excretion of urinary podocytes in patients with type 2 diabetic kidney disease. Biotechnol Biotechnol Equip 29: 139-146, 2015.

14. Zhu B, Wang Y, Jardine M, Jun M, Lv JC, Cass A, Liyanage T, Chen HY, Wang YJ and Perkovic V: Tripterygium preparations for the treatment of CKD: a systematic review and meta-analysis. Am J Kidney Dis 62: 515-530, 2013.

15. Ge Y, Xie H, Li S, Jin B, Hou J, Zhang H, Shi M and Liu Z: Treatment of diabetic nephropathy with Tripterygium wilfordii Hook F extract: a prospective, randomized, controlled clinical trial. J Transl Med 11: 134, 2013.

16. Gao Q, Shen W, Qin W, Zheng C, Zhang M, Zeng C, Wang S, Wang J, Zhu X and Liu Z: Treatment of $\mathrm{db} / \mathrm{db}$ diabetic mice with triptolide: a novel therapy for diabetic nephropathy. Nephrol Dial Transplant 25: 3539-3547, 2010.

17. Huang YR, Wan YG, Sun W, Mao ZM, Zhao Q, Shi XM and Yao J: Effects and mechanisms of multi-glycoside of Tripterygium wilfordii improving glomerular inflammatory injury by regulating p38MAPK signaling activation in diabetic nephropathy rats. Zhongguo Zhong Yao Za Zhi 39: 4102-4109, 2014 (In Chinese).

18. Zhang H, Sun W, Wan Y, Che X, He F, Pu H and Dou C: Preventive effects of multi-glycoside of Tripterygium wilfordii on glomerular lesions in experimental diabetic nephropathy. Zhongguo Zhong Yao Za Zhi 35: 1460-1465, 2010 (In Chinese).

19. Li K and Wang S: Fingerprint chromatogram analysis of extracts from the leaves of Tripterygium wilfordii Hook. F. by high performance liquid chromatography. J Sep Sci 28: 653-657, 2005.

20. Kupchan SM, Court WA, Dailey RG Jr, Gilmore CJ and Bryan RF: Triptolide and tripdiolide, novel antileukemic diterpenoid triepoxides from Tripterygium wilfordii. J Am Chem Soc 94: 7194-7195, 1972.
21. Nakano K, Yoshida C, Furukawa W, Takaishi Y and Shishido K: Terpenoids in transformed root culture of Tripterygium wilfordii. Phytochemistry 49: 1821-1824, 1998.

22. Mao ZM, Shen SM, Wan YG, Sun W, Chen HL, Huang MM, Yang JJ, Wu W, Tang HT and Tang RM: Huangkui capsule attenuates renal fibrosis in diabetic nephropathy rats through regulating oxidative stress and p38MAPK/Akt pathways, compared to $\alpha$-lipoic acid. J Ethnopharmacol 173: 256-265, 2015

23. Wada $\mathbf{J}$ and Makino H: Inflammation and the pathogenesis of diabetic nephropathy. Clin Sci (Lond) 124: 139-152, 2013.

24. Duran-Salgado MB and Rubio-Guerra AF: Diabetic nephropathy and inflammation. World J Diabetes 5: 393-398, 2014.

25. Navarro-González JF and Mora-Fernández C: The role of inflammatory cytokines in diabetic nephropathy. J Am Soc Nephrol 19: 433-442, 2008

26. Wan YG, Sun W, Zhen YJ, Che XY, Pu HP, Wang Y, Li M, Ruan JG and Yan QJ: Multi-glycoside of Tripterygium wilfordii Hook. f. reduces proteinuria through improving podocyte slit diaphragm dysfunction in anti-Thyl.1 glomerulonephritis. J Ethnopharmacol 136: 322-333, 2011.

27. Wan YG, Che XY, Sun W, Huang YR, Meng XJ, Chen HL, Shi XM, Tu Y, Wu W and Liu YL: Low-dose of multi-glycoside of Tripterygium wilfordii Hook. f., a natural regulator of TGF- $\beta 1 /$ Smad signaling activity improves adriamycin induced glomerulosclerosis in vivo. J Ethnopharmacol 151: 1079-1089, 2014

28. Wan YG, Zhao Q, Sun W, Zhang HL, Li M, Wei QX, Wu W, Yue LJ and Wang Q: Contrasting dose-effects of multi-glycoside of Tripterygium wilfordii HOOK. f. on glomerular inflammation and hepatic damage in two types of anti-Thy1.1 glomerulonephritis. J Pharmacol Sci 118: 433-446, 2012.

29. Liu S and Chen ZJ: Expanding role of ubiquitination in NF- $\mathrm{B}$ signaling. Cell Res 21: 6-21, 2011.

30. Nakagawa T, Sato W, Glushakova O, Heinig M, Clarke T, Campbell-Thompson M, Yuzawa Y, Atkinson MA, Johnson RJ and Croker B: Diabetic endothelial nitric oxide synthase knockout mice develop advanced diabetic nephropathy. J Am Soc Nephrol 18: 539-550, 2007.

31. Kodera R, Shikata K, Kataoka HU, Takatsuka T, Miyamoto S, Sasaki M, Kajitani N, Nishishita S, Sarai K, Hirota D, et al: Glucagon-like peptide-1 receptor agonist ameliorates renal injury through its anti-inflammatory action without lowering blood glucose level in a rat model of type 1 diabetes. Diabetologia 54: 965-978, 2011. 